\title{
Spectrophotometric Determination of Fluoxetine by Batch and Flow Injection Methods
}

\author{
Abbas Afrhami, ${ }^{*}$ Tayyebeh Madrakian, and Lida Khalafi \\ Faculty of Chemistry, Bu-Ali-Sina University; Hamadan 65174, Iran. Received June 14, 2006; accepted July 25, 2006
}

\begin{abstract}
A rapid, simple, and accurate spectrophotometric method is presented for the determination of fluoxetine by batch and flow injection analysis methods. The method is based on fluoxetine competitive complexation reaction with phenolphthalein- $\beta$-cyclodextrin $(\mathrm{PHP}-\beta-\mathrm{CD})$ inclusion complex. The increase in the absorbance of the solution at $554 \mathrm{~nm}$ by the addition of fluoxetine was measured. The formation constant for fluoxetin- $\beta$-CD was calculated by non-linear least squares fitting. Fluoxetine can be determined in the range $7.0 \times 10^{-6}-$ $2.4 \times 10^{-4} \mathrm{moll}^{-1}$ and $5.0 \times 10^{-5}-1.0 \times 10^{-2} \mathrm{moll}^{-1}$ by batch and flow methods, respectively. The limit of detection and limit of quantification were respectively $4.13 \times 10^{-6} \mathrm{moll}^{-1}$ and $1.38 \times 10^{-5} \mathrm{moll}^{-1}$ for batch and $2.46 \times 10^{-5} \mathrm{moll}^{-1}$ and $8.22 \times 10^{-5} \mathrm{moll}^{-1}$ for flow method. The sampling rate in flow injection analysis method was $80 \pm 5$ samples $h^{-1}$. The method was applied to the determination of fluoxetine in pharmaceutical formulations and after addition to human urine samples.
\end{abstract}

Key words competitive complexation; fluoxetine determination; inclusion complex; $\beta$-cyclodextrin; flow injection analysis (FIA); pharmaceuticals; UV/VIS

Fluoxetine (FXT) hydrochloride, $N$-methyl-3-phenyl-3-[4(trifluoromethyl)phenoxy] propan-1-amine, (Chart 1) is an important antidepressant drug for the treatment of unipolar mental depression. It enhances serotoninergic neurotransmission through potent and selective inhibition of presynaptic serotonin reuptake. ${ }^{1-3)}$ FXT has been shown to have comparable efficacy to tricyclic antidepressants but with fewer anticholinergic side effects. ${ }^{4}$ Since FXT is a compound of great pharmacological and analytical importance, in recent years there has been an increased interest to develop accurate analytical methods valid for the quantification of FXT in biological and pharmaceutical samples.

Several instrumental methods have been proposed for the determination of FXT in pharmaceutical samples and biological fluids including liquid chromatography, ${ }^{5-7)}$ gas chromatography, ${ }^{8,9)}$ spectrofluorimetry, ${ }^{10,11)}$ and capillary electrophoresis. ${ }^{12,13)}$ Most of the spectrophotometric methods reported for determination of FXT involve difficult and timeconsuming extraction procedures. Giridhar and coworkers determined FXT hydrochloride spectrophotometrically in bulk and in pharmaceutical formulations after extraction of its yellow ion-pair complex by methyl orange and thymol blue with chloroform. ${ }^{14)}$ Starczewska and Mielech reported spectrophotometric determination of FXT and fluvoxamine after extraction of their ion association with chrome azurol S in some organic solvents. ${ }^{15)}$ They also reported spectrophotometric determination of FXT and fluvoxamine based on the extraction of their ion-association with eriochrome cyanine $\mathrm{R}$ in buthanol. ${ }^{16}$

Inclusion compounds are of great interest both in practical application and in fundamental research because they give information about noncovalent intermolecular forces. The most important class of compounds that form inclusion complexes are cyclodextrins (CDs), which are cyclic oligosaccharides composed of six or more glucopyranose units bonded together via 1,4-ether linkages. CDs possess a central cavity into which smaller molecules may "partition," forming noncovalently bonded inclusion compounds.

Owing to their ability to form inclusion complexes, CDs have found extensive applications in many fields including pharmaceutical technology. ${ }^{17,18)}$ Among the properties of a drug that can be improved by complexation with CDs are its aqueous solubility, dissolution rate, absorption, bioavailability, and stability. ${ }^{19)}$

FXT can act as guest to form an inclusion complex with cyclodextrin. ${ }^{20)}$ Formation of $1: 1$ inclusion complex between FXT and methyl- $\beta$-CD was studied at $\mathrm{pH} 7.2$ by spectrofluorimetric method. ${ }^{20)}$

Phenolphthalein (PHP), a typical indicator dye, exhibits the equilibria shown in Chart 2. This compound is used as an acid-base indicator; it is colorless in acidic solutions and red in basic solutions (with the transition occurring around $\mathrm{pH}$ 9). PHP forms a colorless $1: 1$ inclusion complex with $\beta$ $\mathrm{CD}^{21)}$

Koupparis et al. used flow injection gradient technique in spectrophotometric determination of formation constant of the inclusion complex of PHP with $\beta$-CD as $2.4( \pm 0.2) \times 10^{4} 1 \mathrm{~mol}^{-1}$ in carbonate buffer of $\mathrm{pH} 10.5$ and at $23.5^{\circ} \mathrm{C}^{22)}$

The aim of the present study was to develop simple, sensitive, and rapid flow injection analysis (FIA) and batch methods for spectrophotometric determination of FXT based on

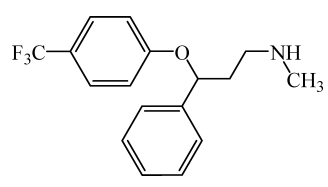

Chart 1

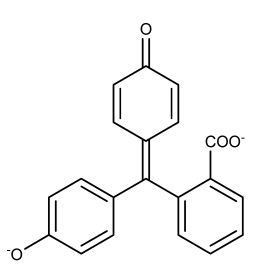

Red

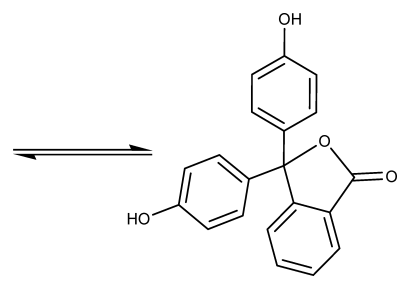

Colorless
Chart 2 


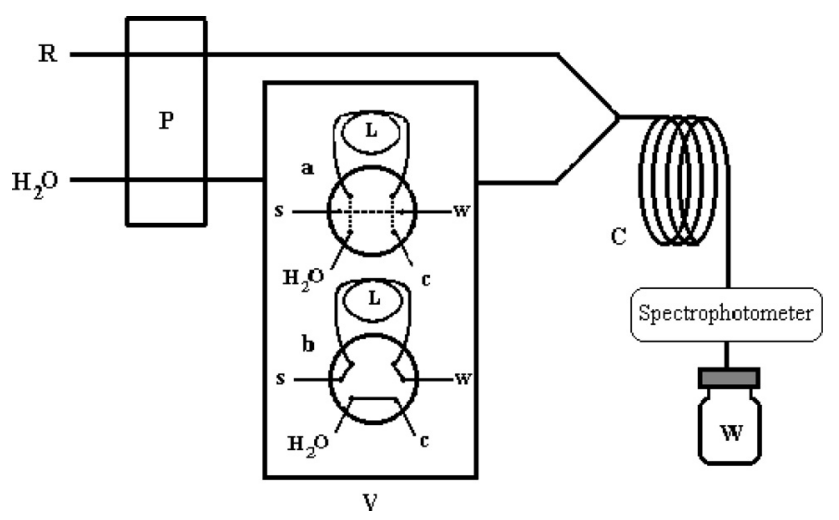

Fig. 1. Schematic Diagram of the Instrumental Set-Up

$\mathrm{R}$, reagents (PHP, $1.2 \times 10^{-4} \mathrm{moll}^{-1} ; \beta-\mathrm{CD}, 2.0 \times 10^{-4} \mathrm{moll}^{-1}$ and carbonate buffer solution, $\mathrm{pH}$ 10.5); $\mathrm{P}$, peristaltic pump; $\mathrm{C}$, coiled reactor; $\mathrm{S}$, sample; $\mathrm{L}$, injection loop; $\mathrm{W}$, waste; V, load-injection valve. Valve positions: (a) sample injection; and (b) loading.

its competitive complexation reaction with $\mathrm{PHP}-\beta$-CD inclusion complex. The spectrophotometric determination of FXT was compared for batch and FIA systems.

\section{Experimental}

Reagents All reagents used were of analytical-reagent grade. Pure FXT hydrochloride was obtained from Lorestan Laboratory (Lorestan, Iran). A $1.5 \times 10^{-3} \mathrm{moll}^{-1}$ stock standard solution of FXT was prepared in water. $\beta$ CD, PHP, and other chemical reagents were purchased from E. Merck and used as received. A $1 \times 10^{-2} \mathrm{moll}^{-1}$ stock solution of $\beta$-CD was prepared in water. All solutions of PHP for the UV-visible spectral measurements were freshly prepared each day. A weighed amount of PHP was first dissolved in a small amount of $\mathrm{NaOH}$ solution and the resultant mixture then diluted with water. Sample dilutions were carried out by taking the appropriate aliquots from the stock solutions followed by dilution with carbonate buffer $(\mathrm{pH}=10.5)$. The total concentrations of prepared buffers were $0.15 \mathrm{moll}^{-1}$.

Apparatus Absorption spectra were obtained by Perkin-Elmer Lambda $45 \mathrm{UV} / \mathrm{Vis}$ spectrophotometer using $1-\mathrm{cm}$ path length quartz cells and the measurements were performed at $25 \pm 0.1^{\circ} \mathrm{C}$.

The flow injection configuration employed is outlined in Fig. 1. Silicon tubing of 0.8 and $1 \mathrm{~mm}$ i.d. was used for the mixing coil and manifold. A four-channel peristaltic pump (Ismatec 404B) was used for propelling the solutions. Samples were injected using a rotating Rheodyne (7725i) valve with a sample loop of $20 \mu \mathrm{l}$. A $20-\mu \mathrm{l}$ flow cell was used.

Recommended Procedure for Determination of FXT by Batch System An appropriate volume of sample containing $3.5 \times 10^{-5}-1.2 \times 10^{-3} \mathrm{mmol}$ FXT was transferred into a $5-\mathrm{ml}$ volumetric flask containing $0.5 \mathrm{ml}$ $1.2 \times 10^{-3} \mathrm{moll}^{-1}$ PHP. Then, $0.2 \mathrm{ml} 5 \times 10^{-3} \mathrm{moll}^{-1} \beta$-CD and $2 \mathrm{ml} \mathrm{pH} 10.5$ carbonate buffer solution were added and the solution was diluted to the mark with distilled water. A portion of the solution was transferred to a 1$\mathrm{cm}$ glass cell to measure its absorbance at $554 \mathrm{~nm}$. A blank solution was also prepared in the same way except that distilled water was used instead of FXT solution and its absorbance was also measured at $554 \mathrm{~nm}$.

Recommended Procedure for Determination of FXT by FIA System The FIA experimental series (Fig. 1) required two separate reagent and distilled water solutions $\left(\mathrm{R}\right.$ and $\mathrm{H}_{2} \mathrm{O}$ ). Solution $\mathrm{R}$ contained optimum concentration of PHP, $\beta-\mathrm{CD}$, and carbonate buffer solution $(\mathrm{pH} 10.5)$ as obtained at batch system. A $20-\mu$ l sample volume containing FXT $\left(5.0 \times 10^{-5}\right.$ $1.0 \times 10^{-2} \mathrm{moll}^{-1}$ ) was injected into the carrier, which was also pumped at a flow rate of $1.6 \mathrm{ml} \mathrm{min}^{-1}$. The absorbance of the mixture was measured and recorded at $554 \mathrm{~nm}$. Under optimum conditions the peak height was proportional to FXT concentration.

\section{Results and Discussion}

Figure 2 shows the absorption spectra for $4.8 \times$ $10^{-5} \mathrm{moll}^{-1} \mathrm{PHP}$ at $\mathrm{pH} 10.5$ in the presence of different concentrations of $\beta$-CD. The absorption spectrum of PHP in the absence of $\beta$-CD shows an absorption band with an absorption maximum at $554 \mathrm{~nm}$. The absorbance decreased at

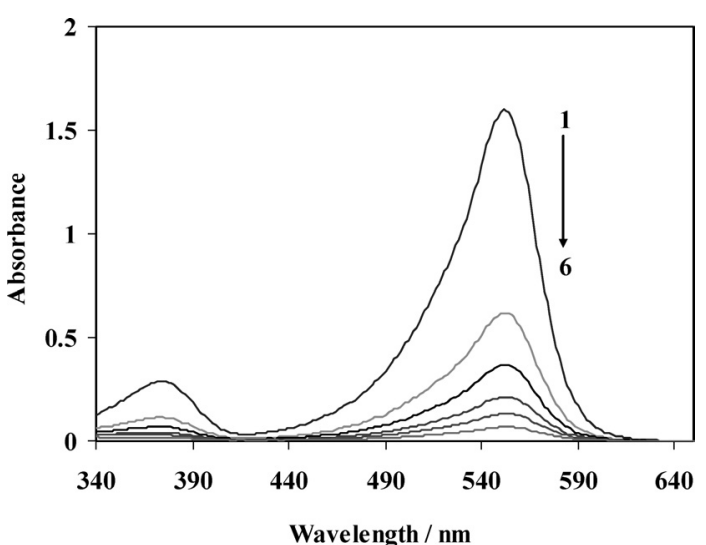

Fig. 2. Absorption Spectra for $4.8 \times 10^{-5} \mathrm{moll}^{-1}$ PHP in the Presence (1) 0.0 , (2) $1.0 \times 10^{-4}$, (3) $2 \times 10^{-4}$, (4) $4 \times 10^{-4}$, (5) $7 \times 10^{-4}$, and (6) $1.0 \times 10^{-3} \mathrm{moll}^{-1}$ of $\beta-\mathrm{CD}$ at $\mathrm{pH} 10.5$

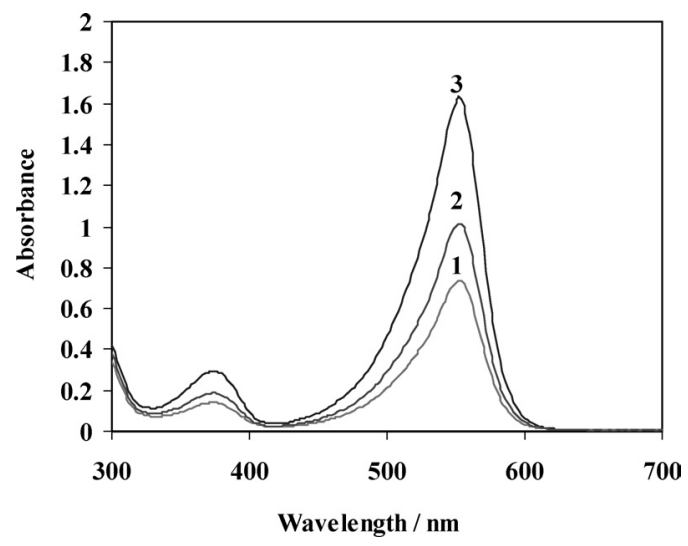

Fig. 3. Absorption Spectra for $4.8 \times 10^{-5} \mathrm{moll}^{-1} \mathrm{PHP}$ at $\mathrm{pH} 10.5$ in the Presence of (1) $1.0 \times 10^{-4} \mathrm{moll}^{-1} \beta$-CD, (2) $1.0 \times 10^{-4} \mathrm{moll}^{-1} \beta$-CD and $2.0 \times 10^{-4} \mathrm{moll}^{-1} \mathrm{FXT}$, and (3) in the Absence of $\beta$-CD and FXT

$554 \mathrm{~nm}$ by increasing $\beta$-CD. When sufficient amount of $\beta$ CD was added to a solution of red (alkaline form) PHP, the color instantly disappeared. As mentioned above, the calculated formation constant for PHP- $\beta$-CD indicates a relatively strong interaction between PHP and $\beta$-CD and it is wellknown that the complex PHP- $\beta$-CD is colorless. When the red, ionized form of PHP is enclosed in $\beta-\mathrm{CD}$, it is forced into its lactone structure without, however, protonating the phenolic groups. That is to say, within the cyclodextrin cavity, the phenolic residues are ionized but the carboxyl group is bound into a lactone. ${ }^{21)}$

Figure 3 shows that by addition FXT to the PHP- $\beta$-CD complex solution the absorbance at $554 \mathrm{~nm}$ increases. This increase in the absorbance is due to the decomposition of $\beta$ CD-PHP inclusion complex by displacement of FXT with PHP. This phenomenon indicates competition of the FXT with PHP in the formation of inclusion complex with $\beta$-CD.

The amount of increase in the absorbance at $554 \mathrm{~nm}$ was found proportional with the FXT concentration over a certain concentration range. Therefore although FXT does not show any absorption band in the visible region, this system could be used for its spectrophotometric determination.

Formation Constant of FXT- $\boldsymbol{\beta}$-CD Complex The competitive complexation equilibra where only the $1: 1$ inclusion complexes of PHP and FXT with $\beta$-CD are formed 
are shown in Eqs. 1 and 2:

$$
\begin{aligned}
& \mathrm{PHP}+\beta-\mathrm{CD} \rightleftharpoons \mathrm{PHP}-\beta-\mathrm{CD} \quad K_{\mathrm{f}, 1}=\frac{[\mathrm{PHP}-\beta-\mathrm{CD}]}{[\mathrm{PHP}][\beta-\mathrm{CD}]} \\
& \mathrm{FXT}+\beta-\mathrm{CD} \rightleftharpoons \mathrm{FXT}-\beta-\mathrm{CD} \quad K_{\mathrm{f}, 2}=\frac{[\mathrm{FXT}-\beta-\mathrm{CD}]}{[\mathrm{FXT}][\beta-\mathrm{CD}]} \\
& C_{\beta-\mathrm{CD}}=[\mathrm{PHP}-\beta-\mathrm{CD}]+[\mathrm{FXT}-\beta-\mathrm{CD}]+[\beta-\mathrm{CD}]
\end{aligned}
$$

The free PHP concentration is obtained from Eq. 4

$$
\begin{aligned}
A & =\left(K_{\mathrm{f}, 1}^{2}-K_{\mathrm{f}, 1} K_{\mathrm{f}, 2}\right)[\mathrm{PHP}]^{3}+\left(C_{\beta-\mathrm{CD}} K_{\mathrm{f}, 1}^{2}+K_{\mathrm{f}, 1}\right. \\
& \left.+\left(2 K_{\mathrm{f}, 1} C_{\mathrm{PHP}}-K_{\mathrm{f}, 1} C_{\beta-\mathrm{CD}}-1\right) K_{\mathrm{f}, 2}\right)[\mathrm{PHP}]^{2}+\left(C_{\beta-\mathrm{CD}} C_{\mathrm{PHP}} K_{\mathrm{f}, 1}\right. \\
& \left.\left.-K_{\mathrm{f}, 1} C_{\mathrm{PHP}}^{2}+2 C_{\mathrm{PHP}}\right) K_{\mathrm{f}, 2}-C_{\mathrm{PHP}} K_{\mathrm{f}, 1}\right)[\mathrm{PHP}]+K_{\mathrm{f}, 2} C_{\mathrm{PHP}}^{2} \\
C_{\mathrm{FXT}} & =\frac{A}{K_{\mathrm{f}, 2}\left(K_{\mathrm{f}, 1} C_{\mathrm{PHP}}[\mathrm{PHP}]-K_{\mathrm{f}, 1}[\mathrm{PHP}]^{2}\right)}
\end{aligned}
$$

where the amount of $K_{\mathrm{f}, 1}$ (formation constant of PHP- $\beta$-CD) is $2.4( \pm 0.2) \times 10^{4} \mathrm{~mol}^{-1}$ in carbonate buffer of $\mathrm{pH} 10.5,{ }^{22)}$ $C_{\beta \text {-CD }}$ and $C_{\mathrm{PHP}}$ are the analytical concentrations of the PHP and $\beta$-CD. Since the PHP- $\beta$-CD complex is colorless, therefore at $\mathrm{pH} 10.5$ the absorbance of the solution is due to free (uncomplexed) PHP. The concentration of free PHP can be calculated from the absorbance of the solution using Beer's law. The absorbance of the solution by addition of different concentrations of FXT was measured. The amount of $K_{\mathrm{f}, 2}$ (the formation constant for FXT- $\beta$-CD complex) was evaluated by non least-squares fits ${ }^{23)}$ of observed absorbances as a function of FXT concentration as $8.2 \times 10^{3} 1 \mathrm{~mol}^{-1}$.

Effect of Chemical Reaction Variables To take full advantage of the procedure for the determination of FXT, the reagent concentrations and reaction conditions must be optimized. The chemical reaction variables including $\beta$-CD concentration, PHP concentration, and $\mathrm{pH}$ were optimized for batch system and the optimum values for batch systems were used in FIA system.

Effect of $\beta$-CD and PHP concentration on the determination of FXT was studied (Fig. 4). In various concentrations of PHP $\left(2.4 \times 10^{-5}\right.$ to $\left.1.6 \times 10^{-4} \mathrm{moll}^{-1}\right)$ and in the presence of different amounts of $\beta$-CD $\left(1.0 \times 10^{-4}\right.$ to $\left.1.0 \times 10^{-3} \mathrm{moll}^{-1}\right)$ absorbance change $(\Delta A)$ at the constant concentration of

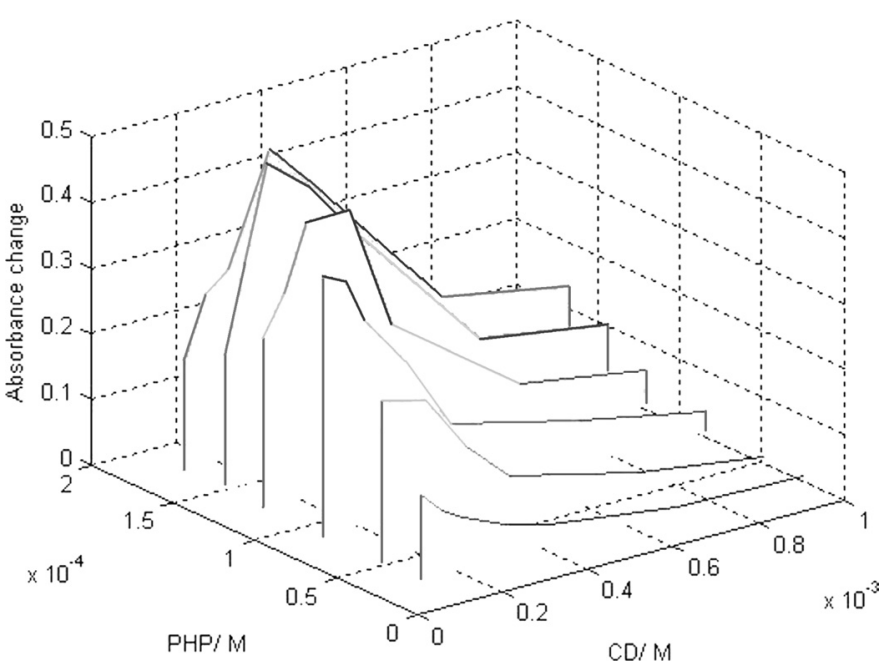

Fig. 4. Absorbance Change for $\beta$-CD-PHP Inclusion Complex at $\mathrm{pH} 10.5$ by the Addition of $2.0 \times 10^{-4}$ mol $1^{-1}$ FXT as a Function of $\beta$-CD and PHP Concentration
FXT $\left(2.0 \times 10^{-4} \mathrm{~mol}^{-1}\right)$ was measured. The maximum $\Delta A$ in this matrix was observed at concentration $2.0 \times 10^{-4} \mathrm{moll}^{-1}$ of $\beta$-CD and $1.2 \times 10^{-4} \mathrm{moll}^{-1}$ PHP. Therefore these values were selected as optimum concentrations of $\beta$-CD and PHP.

Both the complexation reaction of PHP and FXT are $\mathrm{pH}$ dependent. The effect of $\mathrm{pH}$ on the observed $\Delta A$ was studied in the $\mathrm{pH}$ range $8-12$. The results (Fig. 5) showed that $\Delta A$ of the system increased by increasing $\mathrm{pH} \leq 10.5$ and remained nearly constant at higher $\mathrm{pHs}$. Therefore $\mathrm{pH} 10.5$ was selected as optimal.

Effect of Flow Injection Variables In the application of any flow injection method the change of absorbance depends on the residence time of the sample zone in the system, i.e. on the flow rate and reaction coil length. The effects of mixing coil length in the range $50-300 \mathrm{~cm}$ were tested at different flow rates (Fig. 6). Lower lengths produced higher peak heights, but lengths $<150 \mathrm{~cm}$ produced unstable and noisy baseline. This is due to the non-homogeneity of the solution in the flow cell. This effect decreases reproducibility and increase the detection limit of the system. Higher lengths $(>150 \mathrm{~cm})$ resulted in peak broadening and tailing leading to lower sample output. This effect is due to increased dispersion of the sample zone. Therefore a $150-\mathrm{cm}$ reaction coil

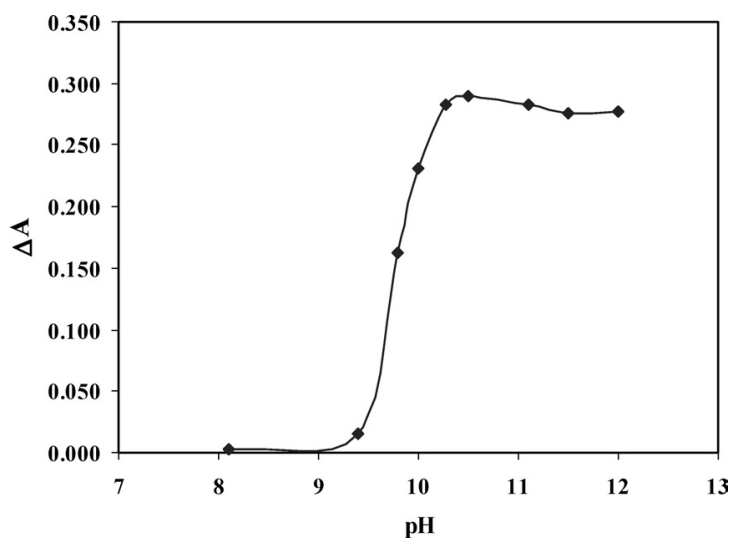

Fig. 5. Effect of $\mathrm{pH}$ on the Absorbance Change of the Solution Conditions: PHP, $4.8 \times 10^{-5} \mathrm{moll}^{-1} ; \beta-\mathrm{CD}, 1.0 \times 10^{-4} \mathrm{moll}^{-1}$; FXT, $2.0 \times 10^{-4}$ $\mathrm{moll}^{-1}$

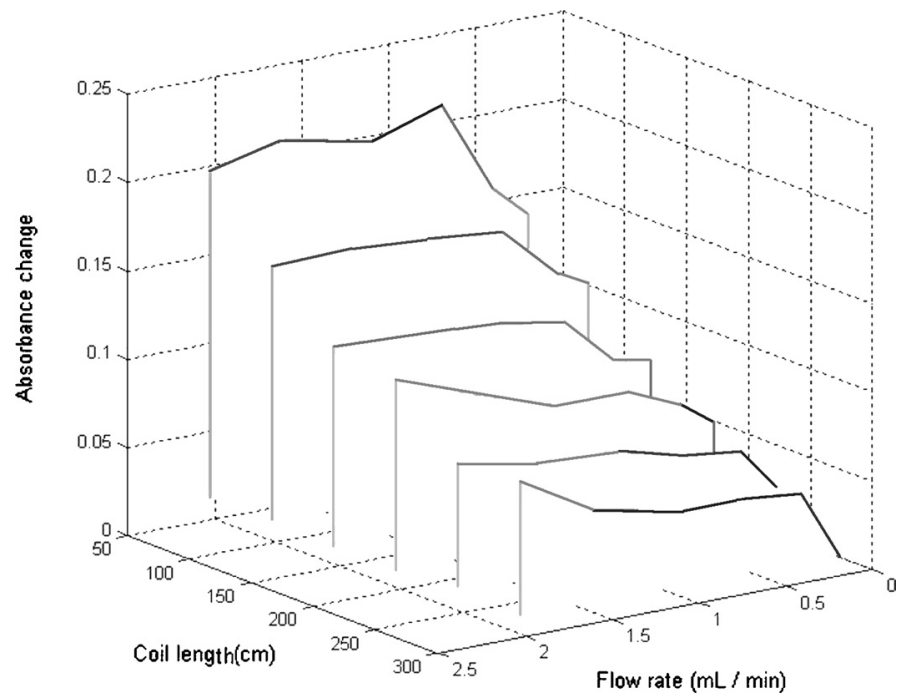

Fig. 6. Absorbance Change for $\beta$-CD-PHP Inclusion Complex at $\mathrm{pH} 10.5$ by Injection of $2.0 \times 10^{-3} \mathrm{moll}^{-1}$ FXT as a Function of Coil Length and Flow Rate 
was selected. The effect of flow rate on peak height was studied over the range $0.2-2.6 \mathrm{ml} \mathrm{min}{ }^{-1}$ for different reaction coils (Fig. 6). The peak height increased $\leq 1.6 \mathrm{ml} \mathrm{min}^{-1}$ then remained more or less constant. Therefore a flow rate of $1.6 \mathrm{ml} \mathrm{min}^{-1}$ was chosen.

Analytical Parameters Batch System: From the measurements made under the optimum conditions described above, the calibration graph was linear in the range $7.0 \times 10^{-6}-2.4 \times 10^{-4} \mathrm{~mol}^{-1}$ of FXT. The calibration equation is $\Delta A=1.561 \times 10^{3} C+0.0041$ with a correlation coefficient of 0.997 , where $C$ is the concentration of FXT in moll ${ }^{-1}$. The limit of detection, defined as $C_{\mathrm{L}}=3 S_{\mathrm{B}} / m{ }^{24)}$ where $C_{\mathrm{L}}, S_{\mathrm{B}}$, and $m$ are the limit of detection, standard deviation of the blank, and slope of the calibration graph, respectively, was $4.13 \times 10^{-6} \mathrm{moll}^{-1}$. The limit of quantification, defined as $C_{\mathrm{LQ}}=10 S_{\mathrm{B}} / \mathrm{m}$ in which $C_{\mathrm{LQ}}$ is the limit of quantification, was $1.38 \times 10^{-5} \mathrm{moll}^{-1}$. To evaluate the precision of the method two different concentrations of standard samples were used and replicate determinations performed. The relative standard deviation for five replicate determinations of $6.0 \times 10^{-5}$ and $1.5 \times 10^{-4} \mathrm{moll}^{-1}$ FXT were 2.01 and $2.56 \%$, respectively.

FIA System: The calibration graph is linear over the range $5.0 \times 10^{-5}-1.0 \times 10^{-2} \mathrm{moll}^{-1}$ of FXT, with the equation: peak height absorbance $(A)=70.2 C+0.0341(C$ is the FXT concentration in $\left.\mathrm{moll}^{-1}\right)$. The resulting graph had a correlation coefficient of 0.999 .

The detection limit $\left(2.46 \times 10^{-5} \mathrm{moll}^{-1}\right)$ was calculated as three times the standard deviation of the peak height measured for five injections. The limit of quantification was $8.22 \times 10^{-5} \mathrm{moll}^{-1}$. The sampling rate was $80 \pm 5$ samples $\mathrm{h}^{-1}$. The relative standard deviation for three replicate determinations of $5.0 \times 10^{-3}, 2.0 \times 10^{-3}, 1.0 \times 10^{-3}$, and $4.0 \times 10^{-4} \mathrm{~mol} \mathrm{l}^{-1}$ FXT were $0.90,1.70,2.0$, and $3.20 \%$, respectively. Figure 7 shows typical responses obtained for different concentrations of FXT.

Analytical Applications To evaluate the analytical applicability of the proposed method the FIA method was applied to the determination of FXT in coated tablet and also after addition to urine samples.

FXT was satisfactorily determined in pharmaceutical preparation (FXT tablets from Arya laboratory, Iran) following the method described in the experimental section. The amount of FXT in the tablet was found as $19.85 \pm 0.4 \mathrm{mg} /$ tablet (manufacturer's value was given as $20.0 \mathrm{mg} /$ tablet $)$. According to the spectral characteristics of the spectrophotometric spectra obtained for these preparations, there is no interference from the excipients.

Two human urine samples were also analyzed by the proposed method. Table 1 summarizes the results obtained with the proposed method for analysis of urine samples spiked with different amounts of FXT. The good obtained recoveries indicate successful applicability of the proposed method for determination of FXT in such samples.

Comparison of the Proposed Method with Other Methods The proposed method was compared with existing methods reported for determination of FXT. The results indicate that the proposed method is more rapid, simpler, and more economical than the existing methods, ${ }^{25,26)}$ provides wider dynamic range, and avoids using toxic organic solvents. ${ }^{15,16)}$

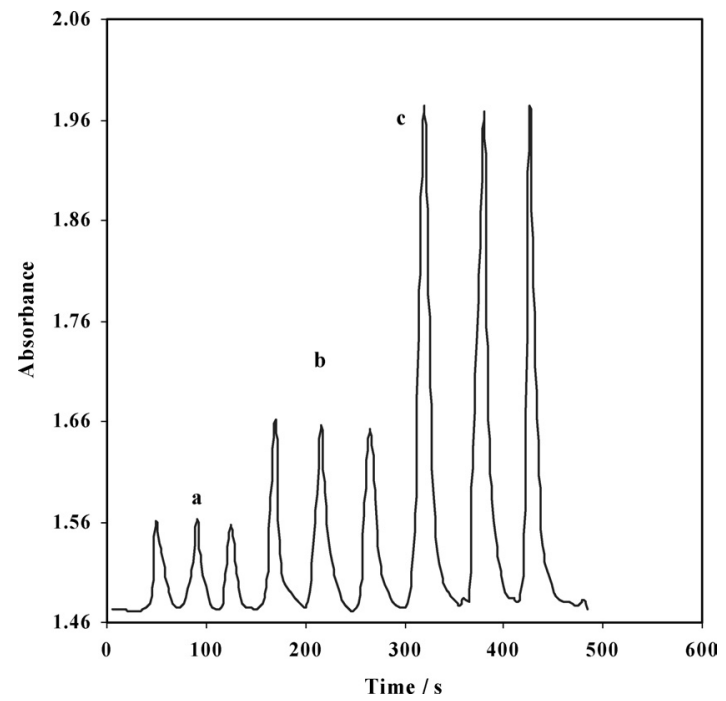

Fig. 7. Typical Responses Obtained for Different Amounts of FXT (a) $1.0 \times 10^{-3} \mathrm{moll}^{-1}$, (b) $2.0 \times 10^{-3} \mathrm{moll}^{-1}$ and (c) $5.0 \times 10^{-3} \mathrm{moll}^{-1}$

Table 1. Determination of FXT after Addition to Urine Samples by the Proposed Method

\begin{tabular}{cccc}
\hline \hline \multirow{2}{*}{ Sample } & \multicolumn{2}{c}{ Amount of FXT $\left(\mathrm{moll}^{-1}\right)$} & $\begin{array}{c}\text { Recovery } \\
(n=3, \%)\end{array}$ \\
\cline { 2 - 4 } & Added & Found & \\
\hline 1 & $5.79 \times 10^{-5}$ & $5.55( \pm 0.4)^{a)} \times 10^{-5}$ & 96.0 \\
2 & $1.74 \times 10^{-4}$ & $1.70( \pm 0.9) \times 10^{-4}$ & 97.0 \\
\hline
\end{tabular}

a) Average of 3 determinations \pm standard deviation.

\section{Conclusion}

The application of competitive complexation reaction to the spectrophotometric determination of FXT in pharmaceutical preparations and human urine samples was described by batch and FIA systems. The inclusion complex between PHP with $\beta$-CD in alkaline solution was used as a spectrophotometric reagent for the determination of FXT. Although the batch system has the advantages of higher sensitivity and lower limit of detection over FIA system, the FIA system has several advantages over the batch system. Simplicity, reliability, reproducibility, time saving, low reagent consumption, need of small sample volume, large dynamic range, and high samples throughput (up to $80 \pm 5$ samples $^{-1}$ ) are important features of the FIA system. The proposed method offers good linearity and precision and can be applied to the analysis of a wide concentration range of FXT in real samples with satisfactory results. The proposed method is rapid, simple, and inexpensive because it requires no sophisticated instrumentation.

This method can also be applied to flow and batch spectrophotometric determination of other drugs that do not absorb in the visible region and can compete with PHP in complexation with $\beta$-CD.

\footnotetext{
References

1) Beufield P., Hell R. C., Lewis S. P., Drugs, 32, 48-52 (1985).

2) Bergstrom R. F., Lemberger L., Farid N. A., Wolen R. L., Br. J. Psychiatry Suppl., 153, 47-50 (1988).

3) Molley B. B., Wong D. T., Fuller R. W., Pharm. News, 1, 6-9 (1994).

4) Cheer S. M., Goa K. L., Drugs, 61, 81-110 (2001).

5) Maya M. T., Domingos C. R., Guerreiro M. T., Morais J. A., J. Pharm.
} 
Biomed. Anal., 23, 989-996 (2000).

6) Raggi M. A., Casamenti G., Mandrioli R., Sabbioni C., Volterra V., J. Pharm. Biomed. Anal., 23, 161-167 (2000).

7) Yilmaz N., Ozkan Y., Ozkan S. A., Biryol I., Aboul-Enein H. Y., J. Liq. Chromatogr. Relat. Technol., 23, 1699-1710 (2000).

8) Lacassie E., Gaulier J. M., Marquet P., Rabatel J. F., Lachatre G., J. Chromatogr. B, 742, 229-238 (2000).

9) Berzas-Nevado J. J., Villasenor-Llerena M. J., Contento-Salcedo A. M., Aguas-Nuevo E., J. Chromatogr. Sci., 38, 200-206 (2000).

10) Martin M. I. G., Perez C. G., Anal. Lett., 30, 2493-2502 (1997).

11) Atmaca S., Pharmazie, 50, 300-301 (1995).

12) Buzinkaiova T., Polonsky J., Electrophoresis, 21, 2839-2841 (2000).

13) Berzas-Nevado J. J., Villasenor-Llerena M. J., Contento-Salcedo A. M., Aguas-Nuevo E., Anal. Chim. Acta, 417, 169-176 (2000).

14) Prabhakar A. H., Patel V. B., Giridhar R., J. Pharm. Biomed. Anal., 20, 427 - 432 (1999)

15) Starczewska B., Mielech K., J. Pharm. Biomed. Anal. 23, 243-247 (2000).

16) Starczewska B., Puzanowska-Tarasiewicz H., Baranowska K., J.
Pharm. Biomed. Anal., 23, 477-481 (2000).

17) Szejtli J., "Cyclodextrin Technology," ed. by Davis J. E. D., Kluwer Academic, Dordrecht, 1988, pp. 186-293.

18) Duchene D., "New Trends in Cyclodextrins and Derivatives," Editions de Santé, Paris, 1992, pp. 351-407.

19) Fromming K. H., Szejtli J., "Cyclodextrins in Pharmacy," ed. by Kluwer, Dordrecht, 1994.

20) Berzas J. J., Alanon A., Lazaro J. A., Talanta, 58, 301-309 (2002).

21) Taguchi K., J. Am. Chem. Soc., 108, 2705-2709 (1986).

22) Georgiou M. E., Georgiou C. A., Koupparis M. A., Anal. Chem., 67, 114-123 (1995).

23) Afkhami A., Khalafi L., Anal. Chim. Acta, 569, 267-274 (2006).

24) Thomsen V., Schatzlein D., Mercuro D., Spectroscopy, 18, 112-114 (2003).

25) Berzas Nevado J. J., Contento Salcedo A. M., Villaseñor Llerena M. J., J. Chromatogr. B, 769, 261-268 (2002).

26) Djordjevic S., Kovacevic I., Miljkovic B., Vuksanovic J., Pokrajac M., Il Farmaco, 60, 345-349 (2005). 